\title{
Multitasking SecB chaperones in bacteria
}

\author{
Ambre Sala, Patricia Bordes and Pierre Genevaux* \\ Laboratoire de Microbiologie et Génétique Moléculaire, Centre National de la Recherche Scientifique, Université Paul Sabatier, Toulouse, France
}

\section{Edited by:}

Salvador Ventura, Universitat

Autònoma de Barcelona, Spain

\section{Reviewed by:}

Claes von Wachenfeldt, Lund

University, Sweden

Julien Brillard, Institut National de la

Recherche Agronomique, France

*Correspondence:

Pierre Genevaux, Laboratoire de Microbiologie et Génétique

Moléculaire, Centre National de la Recherche Scientifique, Université Paul Sabatier, 118 route de Narbonne, 31062 Toulouse Cedex 9, France e-mail: pierre.genevaux@ibcg. biotoul.fr
Protein export in bacteria is facilitated by the canonical SecB chaperone, which binds to unfolded precursor proteins, maintains them in a translocation competent state and specifically cooperates with the translocase motor SecA to ensure their proper targeting to the Sec translocon at the cytoplasmic membrane. Besides its key contribution to the Sec pathway, SecB chaperone tasking is critical for the secretion of the Sec-independent hemebinding protein HasA and actively contributes to the cellular network of chaperones that control general proteostasis in Escherichia coli, as judged by the significant interplay found between SecB and the trigger factor, DnaK and GroEL chaperones. Although SecB is mainly a proteobacterial chaperone associated with the presence of an outer membrane and outer membrane proteins, $\sec B$-like genes are also found in Gram-positive bacteria as well as in certain phages and plasmids, thus suggesting alternative functions. In addition, a SecB-like protein is also present in the major human pathogen Mycobacterium tuberculosis where it specifically controls a stress-responsive toxin-antitoxin system. This review focuses on such very diverse chaperone functions of SecB, both in $E$. coli and in other unrelated bacteria.

Keywords: protein folding and targeting, SecA, DnaK, trigger factor, proteases, toxin-antitoxins

\section{PROTEIN FOLDING AND TARGETING IN BACTERIA}

A major challenge for the cells is to ensure the proper folding and targeting of newly synthesized proteins to the different cellular compartments. Indeed, ongoing protein synthesis in the crowded cellular environment offers a window of opportunities for non-native interactions, which may eventually lead to proteostasis breakdown (Kramer etal., 2009). Therefore, to cope with noxious off pathways in protein biogenesis, cells have evolved universally conserved molecular chaperones and targeting factors, which act co- and/or post-translationally to guide the precise partitioning, localization and folding of newly synthesized proteins (Kramer etal., 2009; Kim et al., 2013).

In bacteria, the folding of newly synthesized proteins is mainly assisted by three highly conserved cytosolic chaperones, namely trigger factor (TF), DnaK/DnaJ/GrpE (DnaKJE), and GroEL/GroES (GroESL; Deuerling et al., 1999; Agashe et al., 2004; Kerner et al., 2005). The ribosome-bound TF is the first chaperone to interact co-translationally with most newly synthesized proteins (Valent et al., 1995). Although the majority of the cytosolic proteins can reach their native state following interaction with TF, a substantial amount of proteins (about 30\%) need further co- and/or post-translational assistance by the downstream DnaKJE and GroESL chaperones (Bukau et al., 2000). Forceful genetic and biochemical analyzes have demonstrated significant overlap and cooperation between these three major chaperones, revealing a dynamic network of chaperones to control intracellular proteostasis (Teter et al., 1999; Genevaux et al., 2004; Calloni et al., 2012).

Targeting of newly synthesized proteins to the bacterial cytoplasmic membrane can occur either co- or posttranslationally. While certain small membrane proteins are targeted post-translationally to the YidC insertase at the inner membrane (Dalbey etal., 2011), most integral membrane proteins as well as some presecretory proteins are targeted cotranslationally by the ribosome-associated RNA-protein complex SRP (Saraogi and Shan, 2014). SRP binds to hydrophobic signalanchor or signal sequence in nascent chains and targets them to the Sec translocon via interaction with its membrane receptor FtsY (Luirink and Sinning, 2004). The majority of presecretory proteins are translocated post-translationally either folded via the twin-arginine translocation (Tat) pathway or in a non-native state via the Sec pathway. The Tat system is known to translocate folded proteins or assembled protein complexes (up to $70 \AA$ in diameter) through the cytoplasmic membrane. Tat substrate proteins possess an amino-terminal signal sequence with a conserved twinarginine motif, which mediates post-translational targeting to the Tat translocon (Palmer and Berks, 2012; Patel et al., 2014). They are often assisted by specific cytosolic chaperones called redox enzyme maturation proteins (REMPs) and by the generic chaperones DnaK and GroEL, which likely prevent their degradation and premature export, and facilitate their assembly and functional interaction with the translocon (Castanie-Cornet et al., 2014).

The Sec translocon is conserved in all three domains of life. Its core is composed of a heterotrimeric membrane complex SecYEG in bacteria and Sec61 $\alpha \beta \gamma$ in eukaryotes (du Plessis et al., 2011). While translocation in the endoplasmic reticulum via the Sec translocon is mainly mediated co-translationally and thus energized by polypeptide chain elongation, Sec translocation across the bacterial plasma membrane preferentially occurs post-translationally and energy is provided by the SecA ATPase motor component (Chatzi et al., 2013). In this case, SecA binds to presecretory proteins with mildly hydrophobic signal sequences, 
targets them to the Sec translocon at the inner membrane via a direct interaction with $\mathrm{SecY}$, and subsequently drives the translocation process by successive cycles of ATP binding and hydrolysis.

Productive interaction with Sec is influenced by the folding rate of the substrate and facilitated by cytosolic chaperones capable of preventing premature folding, aggregation or degradation of precursor proteins (Randall and Hardy, 2002). Accordingly, all three main generic chaperone machines involved in de novo protein folding, namely TF, DnaKJE and GroEL, have been shown to participate at different levels in this process (Castanie-Cornet et al., 2014). Remarkably, most proteobacteria also possess the chaperone $\mathrm{SecB}$, which in addition to its generic chaperone function has the ability to specifically interact with SecA to facilitate post-translational delivery of presecretory proteins to the Sec translocon (Bechtluft et al., 2010). This review describes the Secdependent and Sec-independent cellular functions of SecB, its interplay with other molecular chaperones as well as the distribution of SecB homologs in very diverse bacteria. The role of the recently identified SecB-like proteins in the control of intracellular stress-responsive toxin-antitoxin (TA) systems is also discussed.

\section{SecB AND THE Sec PATHWAY}

$\mathrm{SecB}$ is a homotetrameric chaperone of $69 \mathrm{kDa}$ with a cellular concentration estimated to be between 4 and $20 \mu \mathrm{M}$ in Escherichia coli. $\mathrm{SecB}$ binds co- and/or post-translationally to non-native precursor proteins, maintaining them in competent state for delivery to the Sec translocon via a well-described interaction with its SecA partner (Randall and Hardy, 1995, 2002; Chatzi et al., 2013).

The E. coli $\sec B$ gene was initially identified genetically by selecting for mutants that were defective in the export of a fusion protein composed of the N-terminal part of maltosebinding protein (MBP) preMBP (containing the signal sequence) and $\beta$-galactosidase (Kumamoto and Beckwith, 1983). Further experiments showed that $\sec B$ mutations delayed or blocked the processing of a subset of preproteins and exhibited a synergistic effect with temperature-sensitive alleles of $\sec A$, thus revealing a role for SecB in export (Kumamoto and Beckwith, 1983, 1985). E. coli $\sec B$ mutant strains were initially shown to be defective for growth on rich Luria broth media agar plates, but it later appeared that this phenotype was due to a polar effect on the downstream gpsA gene encoding a glycerol-3-phosphate dehydrogenase involved in phospholipid biosynthesis (Kumamoto and Beckwith, 1985; Shimizu et al., 1997). Deletion of $\operatorname{secB}$ without apparent polarity on $g p s A$ results in a strong cold-sensitive phenotype below $23^{\circ} \mathrm{C}$, a moderate temperature-sensitive phenotype at temperatures above $45^{\circ} \mathrm{C}$ and a hypersensitivity to several antibiotics (Ullers et al., 2007; Table 1). Most of the relevant phenotypes associated with $\sec B$ mutations or SecB overexpression are shown in Table 1. Genetic interactions between $\sec B$ and the various protein localization locus ( $p r l$ ) mutations known to suppress the export defect of sequence signal deficient precursors are also presented (Table 1).

The crystal structures of SecB from Haemophilus influenza (Xu et al., 2000) and E. coli (Dekker et al., 2003) revealed that it forms a tetramer that assembles as a dimer of dimers (Figure 1A).
SecB monomer is composed of four stranded antiparallel $\beta$-sheets (the first two strands being at opposite sides and connected by a cross over loop) and two $\alpha$-helices separated by a helix connecting loop (Figure 1A). SecB dimer is formed via interactions between strands $\beta 1$ and helices $\alpha 1$ of two monomers. The tetramer forms by packing the helices $\alpha 1$ of four monomers in between the eight stranded antiparallel $\beta$-sheets formed by each dimer, mainly via polar interactions. A peptide binding groove was suggested from these structures, lying between the end of the cross over loop and strand $\beta 2$ on one side, and the helix connecting loop and the helix $\alpha 2$ on the other side. This proposed substrate binding region likely contains two subsites: the aromatic, deep subsite 1, and the shallower hydrophobic subsite 2, as presented in Figure 1. Two peptide binding grooves are present on each side of the SecB tetramer, each potentially allowing the binding of $\sim 20$ amino acids-long extended segments. The fact that SecB can bind long fragments of approximately 150 residues in preprotein substrates (Khisty et al., 1995) suggests that these might wrap around the chaperone using several possible routes. Accordingly, electron paramagnetic resonance spectrometry analysis of spin labeled SecB variants in the presence of the physiologic SecB substrate galactose binding protein revealed that in addition to the proposed peptide binding groove, a much larger area of SecB appears to make contact with the substrate (Crane et al., 2006; Figure 1B).

SecB binds to non-native protein substrates with low specificity and high affinity ( $\mathrm{Kd}$ in the nanomolar range), generally in a one to one ratio of tetrameric chaperone to substrate (Randall and Hardy, 1995). SecB binds to regions within the mature part of preprotein substrates and does not specifically recognize signal sequences (Gannon et al., 1989; Liu et al., 1989). Substrate selectivity by $\mathrm{SecB}$ is thought to occur via a kinetic partitioning between binding to the chaperone and folding, which is modulated by the affinity and the folding rate of the substrate protein (Hardy and Randall, 1991). Seminal work performed on the SecB substrate preMBP revealed the appearance of proteolysis resistant conformation of preMBP in the absence of $\mathrm{SecB}$, thus suggesting that binding to $\mathrm{SecB}$ prevents precursor proteins from acquiring a stable tertiary structure incompatible with Sec-dependent translocation (Collier et al., 1988). A single molecule study recently confirmed that binding to SecB maintains preMBP in a molten globule-like state, preventing the formation of stable tertiary interactions (Bechtluft et al., 2007). SecB binding motif was identified by peptide scan of protein substrates as a nine amino acids-long segment enriched in aromatic and basic residues, with acidic residues strongly disfavored. Such motifs statistically occur every 20-30 amino acid residues in both exported and cytosolic proteins, thus suggesting low substrate specificity (Knoblauch et al., 1999).

Several SecB dependent presecretory substrates have been identified in $E$. coli by pulse chase experiments, sequence prediction or following analysis of protein aggregates that accumulate in the absence of the chaperone. This includes 25 presecretory proteins, namely CsgF, DegP, FhuA, FkpA, GBP, LamB, MBP, OmpA, OmpC, OmpF, OmpT, OmpX, OppA, PhoE, TolB, TolC, YagZ, YaiO, YbgF, YcgK, YfaZ, YgiW, YftM, YliI, and YncE (Hayashi and Wu, 1985; Kumamoto and Beckwith, 1985; Kusters et al., 1989; Laminet et al., 
Table 1 | Most relevant phenotypes associated with mutations or overexpression of the $E$. coli SecB chaperone.

\section{SecB Phenotypes ${ }^{a}$}

Single mutation

Cs below $23^{\circ} \mathrm{C}$ and Ts at $46^{\circ} \mathrm{C}$ on LB agar plates ${ }^{(1)}$; sensitive to copper, ethanol, cholate, low $\mathrm{pH}$, dibucaine, triclosan, verapamil, and to several antibiotics, including bacitracin, novobiocin, amoxicillin, carbenicillin, tetracycline, cefaclor, glufosfomycin, ceftazidime, tunicamycin ${ }^{(2)}$; partially resistant to phage $\mathrm{U}^{(3)}$; produces slightly bigger cells ${ }^{(4)}$; and induces synthesis of heat-shock proteins ${ }^{(4,5)}$. Mutation in $\sec B$ with polar effect on the downstream gpsA gene inhibits growth on LB agar plates ${ }^{(6)}$.

Genetic interactions

Mutation in $\sec B$ suppresses erythromycin and rifampin sensitivity of IptE mutants with increased outer-membrane permeability ${ }^{(7)}$; enhances growth and export defects of $\sec A 51$ mutation ${ }^{(4)}$; exacerbates Lon, DnaJ(8) and TF(1) toxicity. Ts, Cs and export defect of $\sec B$ mutation are suppressed by tig mutation ${ }^{(1)}$; Cs is suppressed by lon mutation ${ }^{(8)}$ and by overexpression of $\sigma^{32(9)}$, DnaK/DnaJ ${ }^{(1,10)}, G$ roEL/GroES ${ }^{(10,11)}$, Rv1957(12), SmegB ${ }^{(13)}$, and less efficiently by DnaJ259(8) and SecA(1). Export defect of $\operatorname{secB}$ mutations is partially suppressed by secA853-128 mutation $^{(14)}$. Synthetic lethal with dnaKdnaJ mutation ${ }^{(1)}$ and possibly with mutations in forty-one additional genes, including groEL, the $d s b C$, IOIA, and cpxP genes encoding periplasmic stress proteins and/or chaperones, as well as rp/W encoding for L23, the main chaperone docking site at the ribosomal peptide exit ${ }^{(15)}$. Likely presents positive or negative epistasis with eighty-nine additional mutations in cell envelope biogenesis genes ${ }^{(15)}$.

Protein localization loci

Mutation in secB blocks the phenotypic effects of the pr/C8 (mutation in opdA encoding the cytoplasmic Oligopeptidase A) suppressor of lamB signal sequence mutation ${ }^{(16)}$; inhibits prIA4 ( $\sec Y$ [F286Y, 1408N]) mediated suppression of maltose-binding protein (MBP) signal sequence mutations $^{(17,18)}$ and prlA4 and prlZ1 mediated suppression of LamB signal sequence mutations ${ }^{(19,20)}$. The prlA1001 (secY $\left.[I 90 \mathrm{~N}]\right)$ and prlA1024 $(\sec Y[1408 \mathrm{~N}])$ mutations suppress export deficient maltose-binding protein in the absence of $\mathrm{SecB}^{(17)}$; the prlF1 mutation in the antitoxin gene sohA of the SohA-YhaV toxin-antitoxin system suppresses SecB-dependent accumulation of LamB precursors ${ }^{(21)}$.

Overexpression

Partially suppresses the Ts of the double dnaK tig mutant ${ }^{(22)}$; affects expression of the cytoplasmic response regulator OmpR ${ }^{(23)} ;$ prevents activation of the mycobacterial HigBA1 toxin-antitoxin system expressed in E. coli(12).

a Phenotypes associated with mutation or overexpression of $\operatorname{SecB}$, and genetic interactions between secB and other mutations. Cs and Ts stand for cold-and temperature-sensitive phenotype, respectively.

1 Ullers etal. (2007); ${ }^{2}$ Nichols etal. (2011); ${ }^{3}$ Kumamoto and Beckwith (1985); ${ }^{4}$ Baars et al. (2006); 5 Wild etal. (1993); 6 Shimizu etal. (1997): ${ }^{7}$ Grabowicz etal. (2013): ${ }^{8}$ Sakr etal. (2010); ${ }^{9}$ Altman etal. (1991); ${ }^{10}$ Castanie-Cornet etal. (2014); ${ }^{11}$ Danese etal. (1995); ${ }^{12}$ Bordes etal. (2011); ${ }^{13}$ Sala etal. (2013b); ${ }^{14}$ Mcfarland etal. (1993); ${ }^{15}$ Babu etal. (2011); ${ }^{16}$ Trun and Silhavy (1989); ${ }^{17}$ Francetic etal. (1993); ${ }^{18}$ Derman etal. (1993); ${ }^{19}$ Wei and Stader (1994); ${ }^{20}$ Trun etal. (1988); ${ }^{21}$ Snyder and Silhavy (1992); 22 Ullers etal. (2004); 23 Jin and Inouye (1995).

1991; Powers and Randall, 1995; Baars et al., 2006; Marani et al., 2006). Proteomic analyzes of protein aggregates that accumulate in a $\sec B$ mutant also revealed the presence of a small number of aggregated cytosolic proteins (Baars et al., 2006; Sakr et al., 2010; see SecB Networking).

As stated above, SecB directly targets presecretory proteins to the Sec pathway via its specific interaction with the peripheral ATPase SecA: the motor component of the Sec translocon (Hartl et al., 1990; Figure 2A). SecA is an essential cytosolic protein of $102 \mathrm{kDa}$ with an estimated cellular concentration of $\sim 7 \mu \mathrm{M}$ in E. coli (Kusters et al., 2011; Chatzi et al., 2013). SecA forms a homodimer in solution, is found either soluble or membranebound, and can specifically interact with translating ribosomes mainly via its N-terminal helix (Singh et al., 2014). SecB can interact with both membrane-bound and soluble SecA, albeit with a significantly lower affinity for soluble SecA (1.5 $\mu$ M versus $30 \mathrm{nM}$ Kds, respectively; den Blaauwen et al., 1997). Interaction between $\mathrm{SecB}$ and membrane-bound SecA is further increased in the presence of precursor proteins ( $\mathrm{Kd}$ of $\sim 10 \mathrm{nM}$ ), in order to facilitate the targeting of precursor proteins to the translocon (Fekkes et al., 1997).

Contact regions between SecB and SecA have been studied as well. SecB mutants with amino acid substitutions at positions D20, E24, L75, and E77 that were originally selected on the basis of their export defect (Gannon and Kumamoto, 1993; Kimsey et al., 1995), were later shown to be defective for binding to SecA (Woodbury et al., 2000). Accordingly, the crystal structures of SecB revealed that all these residues localize within the negatively charged surface formed by the $\beta$-sheets on both sides of the tetramer (Figure 1; Xu et al., 2000; Dekker et al., 2003). The main SecB binding site of SecA, which encompasses the last $22 \mathrm{C}$-terminal amino acid of SecA is highly enriched in basic residues and possesses a zinc binding site required for a functional interaction with SecB (Fekkes et al., 1998, 1999). The structure of H. influenzae SecB in complex with the last 24 amino acids of SecA further established such a specific binding occurring mainly through electrostatic interactions, with one SecA C-terminal peptide being bound to each $\beta$-sheet surface on both sides of a SecB tetramer (Zhou and Xu, 2003). 

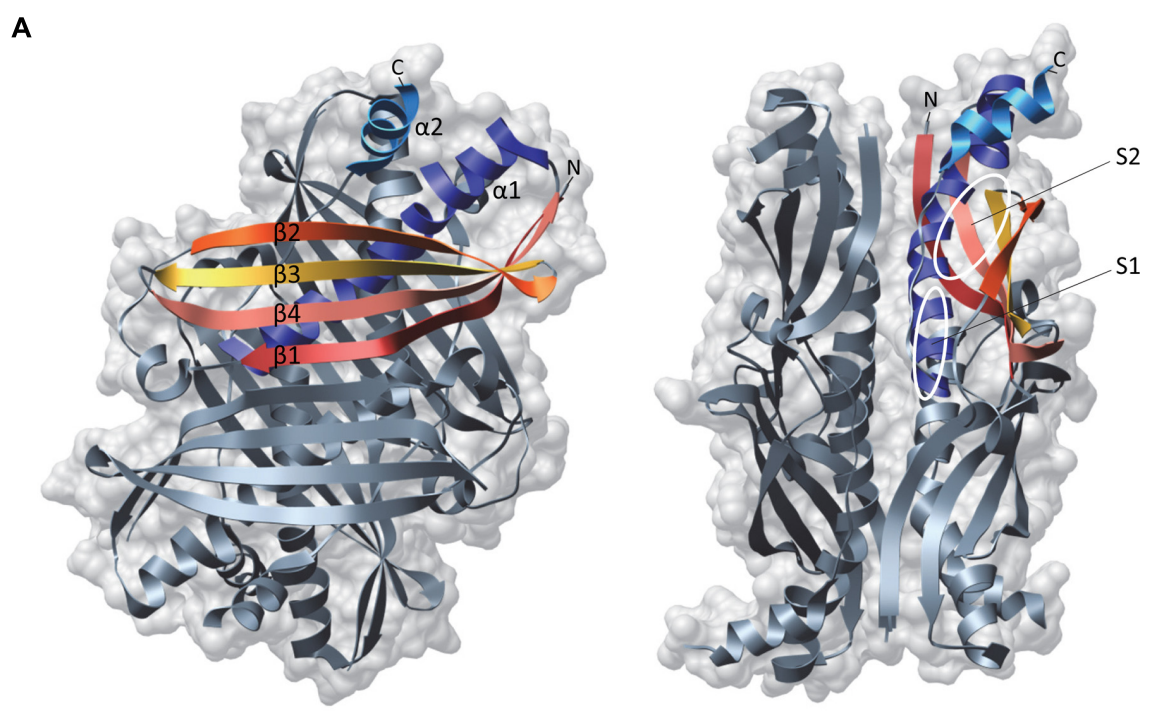

B

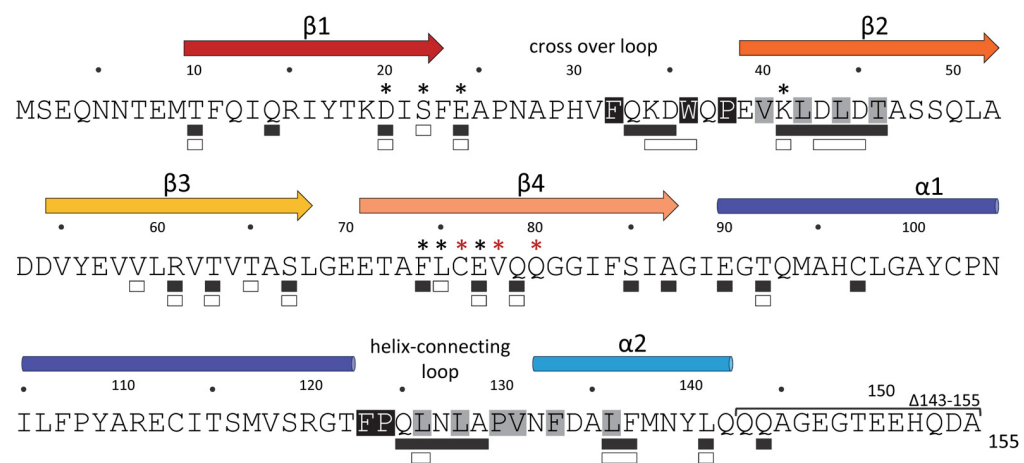

FIGURE 1 |The SecB chaperone. (A) Three-dimensional structure of an Escherichia coli SecB tetramer (1OYN) in front (left) and side (right) views displayed in ribbon and molecular surface using Chimera. Secondary structural elements are colored in both representations as follows: $\beta$-sheet 1 in red, $\beta$-sheet 2 in orange, $\beta$-sheet 3 in yellow, $\beta$-sheet 4 in salmon, $\alpha$-helix 1 in dark blue and $\alpha$-helix 2 in light blue. On the side view, the proposed subsites 1 (S1) and 2 (S2) of interaction with the substrate are indicated. (B) The primary amino acid sequence of $\operatorname{Sec} B$ is annotated with the secondary structural elements on the top with colors corresponding to (A). Residues involved in the interaction with SecA or with the substrate are indicated below the sequence with white or black rectangles, respectively. Black asterisks indicate positions known to alter SecB function in export and red asterisks positions known to trigger aggregation of the protein. The $\mathrm{C}$-terminal deletion 143-155 which alters the interaction with SecA is indicated. The residues predicted as being part of the subsites 1 and 2 of interaction with the substrate are highlighted in black and gray, respectively.
This is consistent with a model in which one SecA dimer binds to one SecB tetramer (Randall et al., 2005). An additional contact site between the two proteins has been described, which consists of the C-terminal $\alpha$-helices of SecB and the N-terminal part of SecA involved in dimerization and ribosome binding (Randall et al., 2004, 2005; Singh et al., 2014). Such interaction was proposed to trigger dissociation of the SecA dimer, thus allowing the opening of a peptide binding groove that would favor substrate transfer from SecB to SecA (Randall et al., 2005). These two surfaces of contact were confirmed by spin-labeling analyzes of SecB upon SecA binding (Crane et al., 2005). Interestingly, this work also showed that the surfaces of SecB that interact with the precursor and with SecA significantly overlap, thus likely facilitating substrate transfer for translocation (Figure 1B; Crane et al., 2005). Efficient transfer of the precursor protein from $\mathrm{SecB}$ to SecA requires both a correct interaction with $\operatorname{SecB}$ and the binding of the functional signal sequence to SecA, which has a strong affinity for signal sequences (Fekkes et al., 1998). To date, the precise mechanism of substrate transfer remains unknown. Once the ATP-dependent translocation process initiates, $\mathrm{SecB}$ is released from the Sec translocon and is now free to initiate a new cycle of binding to precursor proteins (Fekkes et al., 1997). The fact that SecB has the ability to stimulate SecA ATPase activity suggests that it could contribute to the translocation initiation process as well (Miller et al., 2002).

In addition to the post-translational targeting of the SecBprecursor complex to the SecYEG-bound SecA, recent studies suggest that $\mathrm{SecB}$ might be directly recruited to the preformed cytosolic SecA-precursor complex prior to SecA interaction with the protein conducting channel SecYEG (Chatzi et al., 2013; Figure 2A). This model is supported by the recently described 


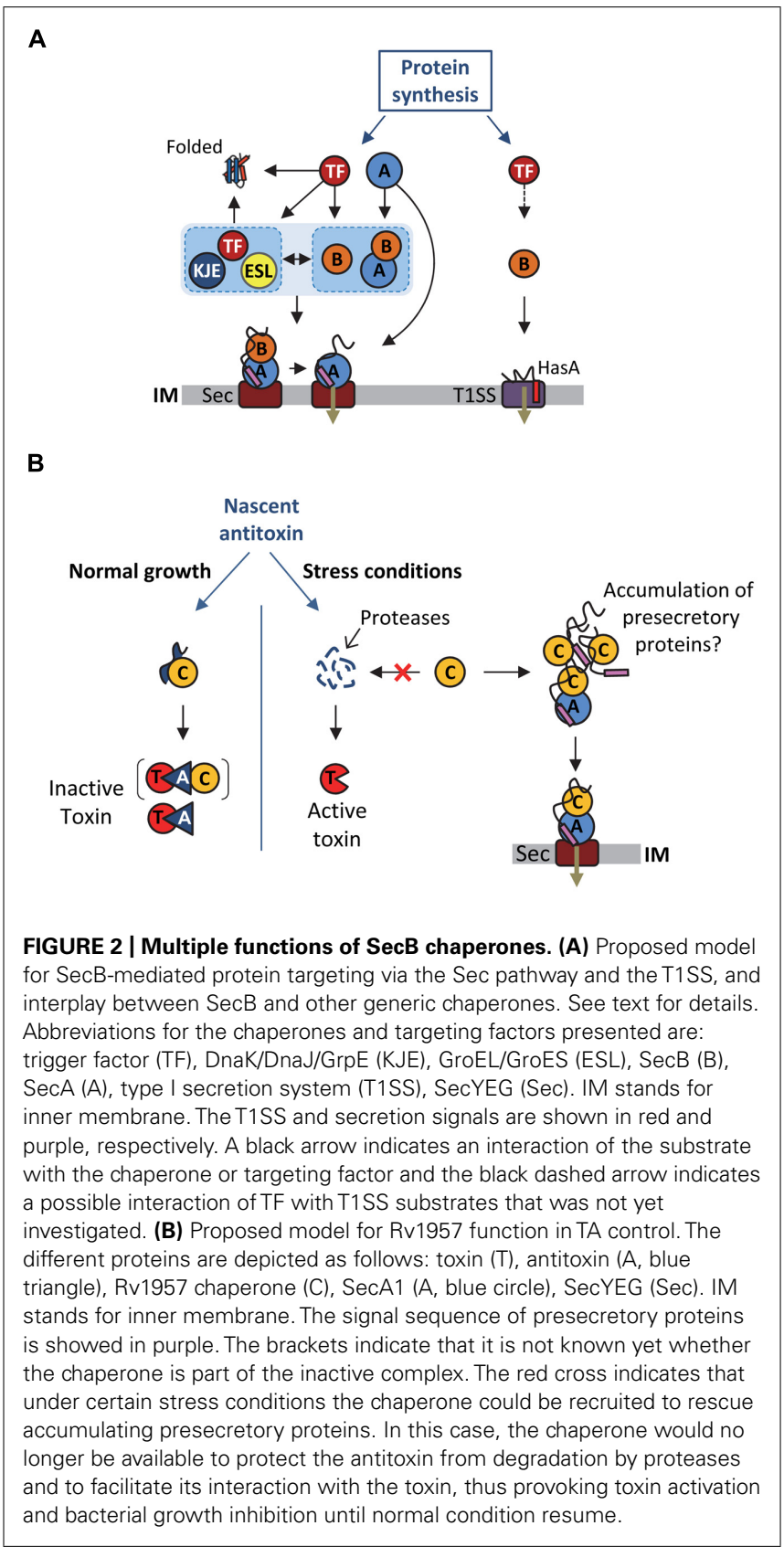

interaction of SecA with the L23 ribosomal protein platform for ribosome interacting factors at the ribosome exit tunnel (Huber et al., 2011; Singh et al., 2014). Together these data further highlight the multifaceted interaction between SecA and SecB, and its key contribution to the selective post-translational targeting of precursor proteins in E. coli.

\section{SecB AND TYPE 1 SECRETION SYSTEMS}

Besides its chaperone function during protein export via the Sec pathway SecB is a key player in the secretion of the small Secindependent HasA hemoprotein $(19.3 \mathrm{kDa})$, which is part of the heme acquisition system of Serratia marcescens (Letoffe et al., 1994). So far, HasA is the only known type 1 secretion system
(T1SS) substrate that is strictly dependent on SecB. The T1SS, which is widespread among Gram-negative bacteria directs the one step translocation of polypeptides across both the inner and outer membranes, directly to the extracellular medium (Delepelaire, 2004). It allows secretion of proteins of diverse sizes (19-800 kDa) and functions (toxins, lipases, heme-binding, or Slayer proteins), which are presumably transported in an unfolded state via a C-terminal uncleaved secretion signal (Delepelaire, 2004; Holland et al., 2005). HasA of Serratia marcescens is secreted by an archetypal T1SS comprising an inner membrane ABC (ATP binding cassette) protein HasD, a periplasmic adaptor HasE, and an outer membrane channel-forming protein of the TolC family, named HasF (Letoffe et al., 1994).

SecB interacts with nascent HasA early during synthesis and holds it in an unfolded conformation competent for productive interaction with the $\mathrm{ABC}$ transporter $\mathrm{HasD}$ at the inner membrane (Figure 2A; Delepelaire and Wandersman, 1998; Debarbieux and Wandersman, 2001). In support of such antifolding activity of $\mathrm{SecB}$, it has been shown that slow folding mutants of HasA are secreted independently of SecB (Wolff et al., 2003). Despite the fact that $\mathrm{SecB}$ allows a functional interaction between the $\mathrm{N}$-terminal region of HasA and $\mathrm{HasD}$, no direct interaction could be detected between SecB and the transporter (Sapriel et al., 2002, 2003; Wolff et al., 2003). Remarkably, point mutations in SecB that are known to affect its interaction with SecA (i.e., mutations D20A, E24A, L75R, and E77V; Figure 1B) exhibited very little or no effect on HasA secretion, thus indicating that $\mathrm{SecB}$ functions independently of SecA in this process. In contrast, SecB mutations affecting its oligomeric state and thus its chaperone function (mutations C76Y and Q80R; Kimsey et al., 1995; Muren et al., 1999) have a very strong effect on HasA secretion (Sapriel et al., 2003), suggesting that substrate binding by $\mathrm{SecB}$ is sufficient in this case. To date, the use of $\mathrm{SecB}$ generic chaperone function by SecAindependent secretion systems has only been shown for HasDEF and it remains to be determined whether other systems require similar assistance by $\mathrm{SecB}$, and to what extent such chaperone redeployment could affect proper functioning of the SecA/SecB cascade in vivo.

\section{SecB NETWORKING}

Significant interplay between $\mathrm{SecB}$ and other major cytosolic chaperones has been described (Castanie-Cornet et al., 2014; Figure 2A). The functional cooperation and/or overlap, as well as the strong genetic interactions observed between SecB, TF and DnaKJE suggest a key role for SecB as part of the chaperone network that orchestrates proper protein folding and targeting in $E$. coli. Albeit significantly less studied, a discrete link between SecB and the chaperonin GroEL has also been shown in some cases. In this part, we describe the intricate relationship between SecB and these main chaperones and discuss how SecB chaperone tasking contributes to such proteostasis network.

\section{SecB AND THE RIBOSOME-BOUND TRIGGER FACTOR CHAPERONE}

The TF chaperone interacts with most newly synthesized polypeptides in E. coli (Valent et al., 1995). It is believed that about 70\% of the E. coli cytosolic proteins interacting with TF reach their native state without further assistance (Deuerling et al., 1999; Teter 
et al., 1999). TF specifically binds to the ribosomal protein L23 in the vicinity of the polypeptide exit tunnel and cycles on and off the ribosome in an ATP-independent manner (Kramer et al., 2002; Ferbitz et al., 2004; Genevaux et al., 2004). Following release from the ribosome, TF can stay bound to elongating polypeptides and facilitate substrate transfer to downstream chaperones or possibly to the Sec translocon (Crooke et al., 1988b; Kaiser et al., 2006; Raine et al., 2006; Hoffmann et al., 2010; Saio et al., 2014). TF can delay the folding of large proteins and exhibits unfolding activity (Agashe et al., 2004; Hoffmann et al., 2012; O'Brien et al., 2012), which may facilitate targeting of presecretory proteins to the Sec translocon, as observed for SecB. TF interacts with outer membrane proteins (OMPs) and several OMPs and periplasmic proteins are significantly decreased in the absence of TF (Oh et al., 2011). Remarkably, a substantial number of these exported subtrates is shared between SecB and TF: this includes precursors of OmpA, OmpC, OmpF, LamB, PhoE, TolC, DegP, FkpA, OppA, Bla, and MBP (Castanie-Cornet et al., 2014). Yet, in contrast with $\mathrm{SecB}$, a direct role for TF in stabilizing translocation competent precursors has only been shown for proOmpA and in this case, deletion of the tig gene encoding TF exhibited no significant defect on proOmpA processing (Crooke and Wickner, 1987; Crooke et al., 1988a,b). Instead, tig mutation was shown to accelerate translocation of several known SecB substrates, namely OmpA, OmpC, and OmpF (Lill et al., 1988; Guthrie and Wickner, 1990; Lee and Bernstein, 2002; Genevaux et al., 2004; Ullers et al., 2007) and to fully suppress both cold-sensitive and temperaturesensitive phenotypes of a $\sec B$ null strain (Table 1; Guthrie and Wickner, 1990; Lee and Bernstein, 2002; Genevaux et al., 2004; Ullers et al., 2007). These data suggest that ribosome-bound TF could facilitate post-translational targeting of precursors by maintaining them competent either for binding to membrane-bound SecA (Gouridis et al., 2009) or for transfer to SecB, DnaKJE, or GroESL (Figure 2A; see subsections below). The fact that both TF and SecA bind to L23 at the ribosomal polypeptide exit suggests that TF could either cooperate with SecA or prevent unproductive SecA binding to precursors that first need to transit via $\mathrm{SecB}$ (Figure 2; Karamyshev and Johnson, 2005; Huber et al., 2011; Singh et al., 2014). Although more work is needed to shed light on such possible interplays between SecA, TF and SecB, it is important to note that both $\operatorname{secB}$ and $r p l W$ (the gene encoding L23) mutations likely synergize in vivo, further supporting an important role for SecB in this process (Table 1).

\section{SecB AND THE DnaKJE CHAPERONE MACHINE}

The ATP-dependent chaperone DnaK of E. coli is a wellcharacterized member of the Hsp70 chaperone family. It is an abundant cytosolic chaperone expressed constitutively and induced in response to different stresses (Genevaux et al., 2007). The DnaK chaperone cycle is tightly regulated by essential co-chaperones: (i) the DnaJ (Hsp40) co-chaperone family members that stimulate DnaK's weak ATP activity and facilitate substrate delivery to DnaK, and (ii) the nucleotide exchange factor GrpE, which mediates the dissociation of ADP and the subsequent binding of a new ATP that triggers substrate release from DnaK (Liberek et al., 1991; Harrison et al., 1997; Brehmer et al., 2001). DnaK preferentially interacts with short extended hydrophobic polypeptide sequences accessible during de novo protein folding, translocation through biological membranes, during stress or within native protein complexes (Rudiger et al., 1997). In agreement with such a variety of potential interactors, the recently described in vivo interactome of DnaK obtained in the presence of SecB revealed that DnaK interacts with more than six hundred E. coli proteins at $37^{\circ} \mathrm{C}$, including cytosolic $(\sim 80 \%)$, inner membrane $(\sim 11 \%)$, outer membrane $(\sim 3 \%)$ and periplasmic proteins ( $3 \%$; Calloni et al., 2012).

Most of our current knowledge concerning DnaKJE's contribution to the Sec pathway originates from studies concerning $\sec B$ mutants and/or SecB substrates (Castanie-Cornet et al., 2014). Indeed, it has been shown that export of the SecB substrates OmpA, OmpC, and OmpF strongly relies on DnaK when protein translocation is compromised (Qi et al., 2002), and that overexpression of DnaKJ suppresses both the cold-sensitive phenotype of a $\sec B$ null strain and the export defect of the SecB-dependent substrates LamB and MBP (Wild et al., 1992; Ullers et al., 2007; Castanie-Cornet et al., 2014). Although, export of both LamB and MBP is not affected by a dnaK mutation (Wild et al., 1992), depletion of DnaKJ in the absence of SecB showed a further decrease in the processing of these proteins and a robust accumulation of protein aggregates in the E. coli cytoplasm (Wild et al., 1992; Ullers et al., 2007). These aggregated proteins include known DnaK substrates and several OMPs (i.e., OmpA, OmpC, OmpX, and PhoE) previously known as SecB substrates (Ullers et al., 2007). Such a major overlap between these two chaperones is further supported by the fact that $\mathrm{SecB}$ substrates were recently identified as bona fide DnaK interactors in vivo (Calloni et al., 2012). This includes the OMPs OmpA, OmpC, OmpF, OmpT and OmpX, and the periplasmic proteins OppA and DegP. Accordingly, peptide binding scans revealed that $\mathrm{SecB}$ and DnaK share many potential binding sites in polypeptide substrates and could interact with similar regions within protein (Knoblauch etal., 1999). These data are in complete agreement with the fact that mutations in $\operatorname{secB}$ and $d n a K$ (or dnaJ) exhibit synthetic lethality (Table 1), and that expression of DnaK is upregulated in the absence of $\mathrm{SecB}$, and reciprocally (Muller, 1996; Ullers et al., 2007). These data also suggest that both chaperones could work in concert to assist the posttranslational translocation of certain Sec substrates (Sakr et al., 2010). The physical interaction recently found between SecB and DnaK in vivo is in agreement with such hypothesis (Calloni et al., 2012).

\section{SecB AND THE TF/DnaK PATHWAY FOR CYTOSOLIC PROTEIN FOLDING}

In addition to protein export, a role for $\mathrm{Sec} B$ in rescuing cytosolic protein folding has been proposed. Such a SecB function has emerged from studies generally focusing on both TF and DnaK chaperones. Indeed, it has been shown that SecB overexpression efficiently rescues the severe growth defect of a chaperone-deficient strain carrying both $d n a K$ and tig mutations, and suppresses the DnaK/TF-dependent accumulation of aggregated cytosolic proteins (Ullers et al., 2004). In vitro cross-linking experiments further showed that $\mathrm{SecB}$ is indeed capable of interacting co- and/or post-translationally with nascent RpoB in the absence of both chaperones. Such a possible SecB function is further supported by the fact that (i) SecB has preference for 
unstructured stretches of polypeptides that are not specifically found in exported proteins (Knoblauch et al., 1999), (ii) SecB prevents luciferase aggregation and cooperates with DnaKJE in the refolding of luciferase in vitro (Knoblauch et al., 1999), and (iii) cytosolic proteins can be isolated from aggregated protein fractions in both $\operatorname{secB}$ and $\sec B$ lon mutant strains (Baars et al., 2006; Sakr et al., 2010). More work is warranted to elucidate whether SecB indeed has cytosolic protein substrates in vivo. Of note, the SecBlike chaperone Rv1957 in Mycobacterium tuberculosis was shown to directly assist the folding of a cytosolic antitoxin, arguing for such possible SecB function in other bacteria (see part below).

\section{SecB AND THE CHAPERONIN GroESL}

The third main molecular chaperone potentially linked to SecB in E. coli is the chaperonin GroESL. The ATP-dependent chaperonin GroEL is a well-characterized member of the Hsp60 chaperone family. Together with its co-chaperone GroES (Hsp10), it provides both a protected environment and a functional assistance to polypeptides generally up to $60 \mathrm{kDa}$. GroEL forms a barrel-shaped complex composed of two heptameric rings assembled back-toback (Saibil et al., 2013). The GroEL folding cavity can be closed by a seven GroES co-chaperone lid, which allows confinement of the polypeptide. It is believed that GroESL interacts with more than $10 \%$ of the E. coli cytosolic proteins, including aggregation-prone proteins that are strictly chaperonin-dependent for their folding in vivo (Kerner et al., 2005).

Although poorly investigated, a direct involvement of GroESL in the Sec pathway has been observed and several SecB substrates have been shown to interact with or to be processed by GroEL (Kusukawa et al., 1989; Lecker et al., 1989; Phillips and Silhavy, 1990). Remarkably, five known SecB substrates were recently identified as GroEL interactors in vivo. These include three OMPs, namely OmpA, OmpC and OmpF, and two periplasmic proteins OppA and YncE (Watanabe et al., 1988; Kerner et al., 2005; Baars et al., 2006). In addition, GroEL was previously shown to interact with prePhoE and proOmpA in vitro, and to stabilize proOmpA for translocation (Kusukawa et al., 1989; Lecker et al., 1989). Although groESL mutations exhibit no apparent effect on proOmpA and proOmpF processing, overexpression of GroESL efficiently rescues the cold-sensitive phenotype of a $\sec B$ null strain (unpublished data). The fact that endogenous SecB level also increases in strains with impaired GroESL is in agreement with such findings (Muller, 1996). Together these data suggest that GroESL may actively contribute to the Sec-dependent export process, perhaps rescuing $\mathrm{SecB}$ substrates under certain stresses or even cooperate with $\mathrm{SecB}$ to facilitate their transfer to SecA, as proposed for TF and DnaK.

\section{SecB-LIKE CHAPERONES AND TOXIN-ANTITOXIN SYSTEMS}

As stated above, SecB is usually found in proteobacteria. Yet, some SecB-like sequences are also found in other taxonomic groups, including Gram-positive bacteria (Sala et al., 2013b). The major human pathogen $M$. tuberculosis also encodes a SecB-like protein, namely Rv1957, which shares 19\% amino acid sequence identity with the E. coli SecB. The fact that mycobacteria have a well-defined and characteristic outer membrane, named the mycomembrane, with a significant number of predicted OMPs suggests that these bacteria could make use of such SecB chaperone function for the targeting of their OMPs to the Sec translocon (Zuber et al., 2008; Mah et al., 2010). Previous work showed that Rv1957 can replace SecB export function in E. coli, partially restoring the processing of both proOmpA and preMBP, and complement the cold-sensitive phenotype of a $\sec B$ mutant strain (Table 1). In vitro, Rv1957 also forms a tetramer in solution and efficiently prevents aggregation of the known E. coli SecB substrate proOmpC at a level comparable to that of SecB (Bordes et al., 2011). These results strongly suggest that Rv1957 could act as a bona fide SecB chaperone to assist protein export in M. tuberculosis.

In contrast with E. coli SecB, the Rv1957 encoding gene is clustered together with genes that are part of a stress-responsive type II TA system related to the HigBA family (Host Inhibition of Growth; Gupta, 2009; Ramage et al., 2009; Bordes et al., 2011; Sala et al., 2013a,b; Schuessler et al., 2013). Type II TA systems are genetic modules composed of a stable toxin and a less stable antitoxin, which interact together to form a complex in which the toxin is inactive (Gerdes et al., 2005; Goeders and Van Melderen, 2014). Under specific stress condition, the antitoxin is degraded by activated proteases, provoking the release of the active toxin, which will then act on its intracellular targets. Toxins from type II TA generally target essential cellular functions, such as translation or replication, resulting in growth inhibition. Modulation of bacterial growth by TA systems in response to environmental insults likely favors adaptation to stress (Lewis, 2010; Yamaguchi and Inouye, 2011). Remarkably, the SecB-like chaperone Rv1957 from M. tuberculosis specifically controls the inhibition of the HigBA TA system (Figure 2B). Indeed, Rv1957 interacts directly with the HigA antitoxin and protects it from both aggregation and degradation by proteases, thus facilitating its folding and subsequent interaction with the toxin. This chaperone function is necessary for the efficient inhibition of the toxin by the antagonistic antitoxin (Bordes et al., 2011). Such a tripartite system, named TAC for toxin-antitoxin-chaperone, is the first example of a TA system controlled by a molecular chaperone.

The hypothetic dual role of Rv1957 both as a generic chaperone potentially assisting protein export and as a specialized chaperone controlling a bacterial growth tuning system raises the question of a possible link between these two functions under certain conditions (Figure 2B). An attractive hypothesis is that in case of a compromised translocon accumulation of preproteins could compete with the antitoxin for Rv1957 binding, resulting in antitoxin degradation and subsequent toxin activation. In this model, the SecB-like chaperone would thus function as a molecular sentinel to watch over protein export. The fact that the presence of a $\sec B$ open reading frame associated with TA modules is not unique to M. tuberculosis or to mycobacteria (Sala et al., 2013b) indicates that such a mechanism might be conserved (see part below).

\section{TAXONOMIC DISTRIBUTION OF SOLITARY AND TA-ASSOCIATED SecB}

It has been proposed that SecB appeared in the last common ancestor of $\alpha-, \beta$-, and $\gamma$-proteobacteria and that its conservation is linked to the presence of an outer membrane, and 
thus an increased need in protein export (van der Sluis and Driessen, 2006). Nevertheless, analysis of the taxonomic repartition of PF02556, the Pfam domain characterizing SecB sequences (http://pfam.sanger.ac.uk/) in a set of 1631 complete and cured bacterial genomes revealed the presence of this domain in seven groups outside proteobacteria. Noticeably, these groups are mainly composed of diderm bacteria, except from the Firmicutes phylum (Figure 3A) and in most cases, SecB sequences occur at low frequency when compared to the total number of genomes. This is in sharp contrast with $\alpha$-, $\beta$-, and $\gamma$-proteobacteria, where most of the genomes contain at least one SecB sequence (Sala et al., 2013b; Figure 3A).
A subset of SecB genes, representing approximately $7.5 \%$ of the total number of SecB sequences (52/688), are associated with genes encoding TA systems (or in some cases antitoxin genes alone), as observed for the TAC system of M. tuberculosis. This suggests that these putative $\mathrm{SecB}$ chaperones might function in the specific control of their cognate TA systems in a manner comparable to that of Rv1957 (Bordes et al., 2011; Sala et al., 2013b). When SecB sequences are present in groups outside of $\alpha$-, $\beta$-, and $\gamma$ proteobacteria, they seem to preferentially associate with a TA system $(63 \%, 44 / 70)$. In this case, the vast majority of the genomes (>90\%) do not possess an additional copy of solitary SecB. Interestingly, TA systems associated with SecB sequences often belong

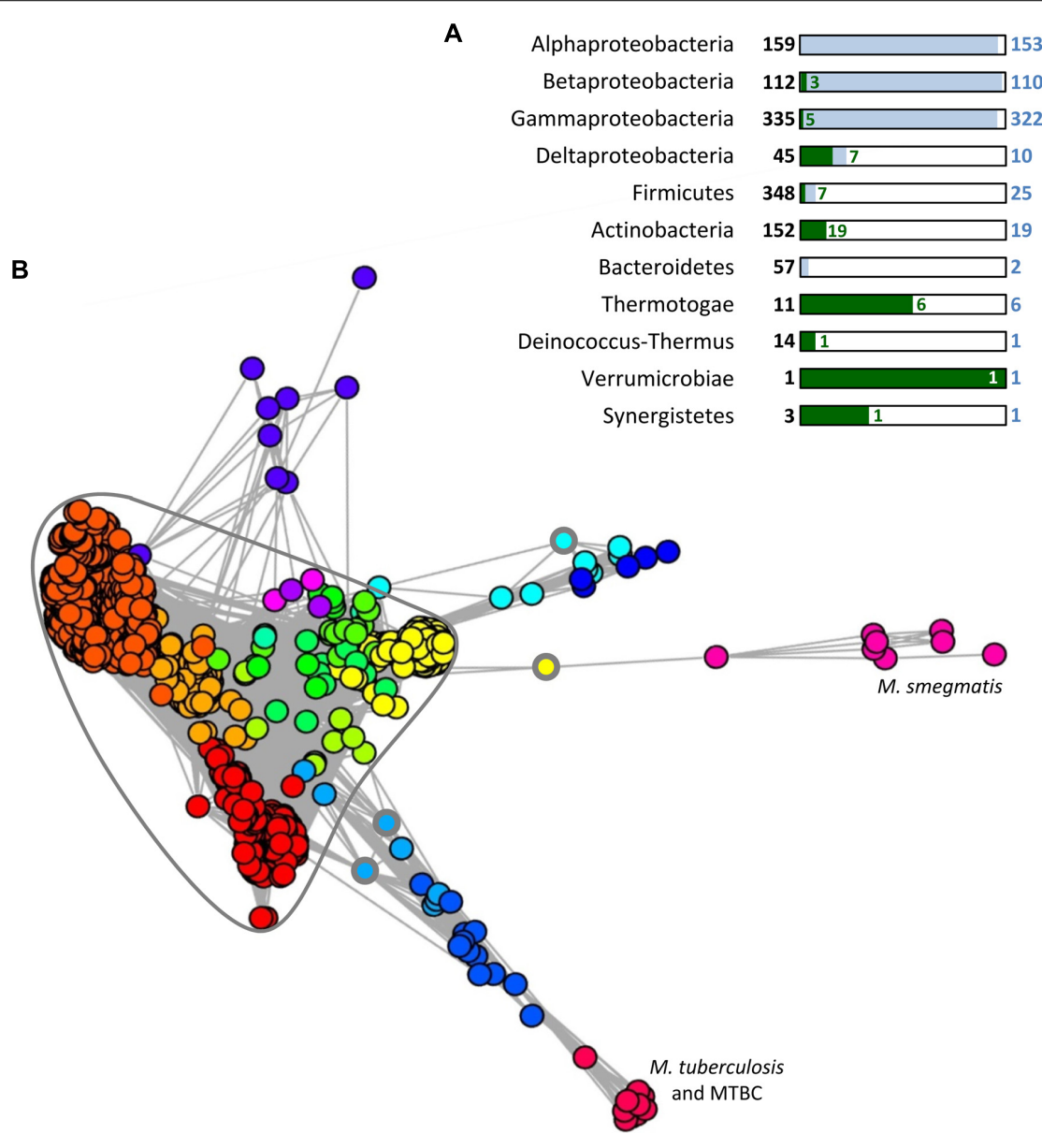

FIGURE 3 | Distribution and conservation of SecB sequences in bacteria. (A) Taxonomic distribution of SecB sequences. A number of 1631 complete and cured bacterial genomes (local database) were analyzed for the presence of SecB sequence by searching for (i) the signature of the PF02556 domain using RPS-BLAST with as threshold an e-value of $10^{-5}$, a score of 35 and a query coverage of $40 \%$, or (ii) TAC (or AC) chaperones potentially not identified by the first request, using an approach described previously (Sala et al., 2013b). This approach allowed the identification of $688 \mathrm{SecB}$ sequences. For each taxonomic group containing $\operatorname{Sec} B$ sequences, the total number of genomes is given in bold characters and represented by a black frame. The number of genomes containing one or more SecB sequences is given in blue and represented by blue rectangles, and the number of genomes containing TAC or AC systems is depicted in green. (B) MCL (Markov Clustering) analysis of the 1981 bacterial sequences contained in the PF02256 conserved domain plus 33 sequences identified previously (Sala et al., 2013b) as TAC chaperones that are absent from the PF02556. A graph was built in which nodes (Circles) correspond to protein sequences and weighted edges represent the BLASTP log(e-value) obtained between a pair of proteins. In this case, the homology relationship was inferred when an e-value less than or equal to $10^{-5}$ was observed between two sequences. Partitioning of this graph into communities of highly connected nodes was performed using the $\mathrm{MCL}$ program, with an inflat parameter of 1.5. This resulted in 18 groups of SecB sequences, represented by the different colors assigned to the circles in the graph. The highly connected core containing almost all solitary SecB sequences discussed in the text is circled in gray. The three circles with a thick gray line indicate the solitary $\operatorname{Sec} B$ sequences found outside of the $\mathrm{SecB}$ conservation core. MTBC stands for $M$. tuberculosis complex species. 
to different families of toxins and/or antitoxins, strongly suggesting that the event of association of a SecB encoding gene with a TA module is a widespread mechanism that occurred several times during evolution (Sala et al., 2013b). The possible involvement of these SecB chaperones in Sec-dependent protein export remains to be determined.

Further analysis on all the bacterial sequences available on the Pfam server for the PF02556 conserved domain (i.e., from both complete and in progress genomes) was performed to study the homology links between $\mathrm{SecB}$ sequences using a graph partitioning approach. This revealed that solitary SecB sequences are grouped together in a highly connected core, reflecting a high level of conservation (Figure 3B). In this core, several SecB communities (corresponding to the different colors) are well-defined and generally correspond to the taxonomy: the red family contains mainly $\alpha$-proteobacterial sequences, the dark orange mainly $\gamma$-proteobacterial sequences and the light orange mainly $\beta$-proteobacterial sequences. Another clearly defined group that emerges from this core, in yellow, contains mainly sequences from Streptococcus pneumoniae strains $(158 / 196)$. The other communities within the core are poorly defined and mainly correspond to other Firmicutes sequences. Most of the TAC (or AC) chaperones are grouped in eight different communities, which seem to have diverged from the solitary SecB core from distinct origins (Figure 3B). The light orange group within the core also contains four TA-associated $\mathrm{SecB}$ sequences from $\delta$-proteobacteria, thus strongly suggesting a common evolutionary history between TAC chaperones and canonical solitary SecB (Sala etal., 2013b). Yet, in sharp contrast with solitary $\mathrm{SecB}$, the groups of TAC chaperones do not follow the taxonomy and most of them are comprised in regions containing horizontal gene transfer signatures, as it is the case for classical TA systems (Makarova et al., 2009; Sala et al., 2013b).

Paralogs of SecA, SecE, SecY, and SecG are found in Actinobacteria and Firmicutes, either forming a parallel pathway with a dedicated translocon or exploiting the generic Sec translocon to export a specific set of substrates, as it is the case for SecA2 in mycobacteria (Rigel et al., 2009; Sullivan et al., 2012). Interestingly, among the 1631 complete bacterial genomes analyzed 26 of them are predicted to have more than one solitary SecB sequence (up to three in Acetobacter pasteurianus IFO 3283-01$42 \mathrm{C}$ ), eight genomes contain both a solitary and a TA associated $\mathrm{SecB}$, one genome contains two solitary and one TA associated SecB, and two genomes contain two TAC or AC. These additional SecB sequences could function as specialized chaperones for the control of TA systems or for the export of specific substrates, or as generic chaperone induced in response to certain stress conditions. Interestingly, single deletion of $\sec B 1$ or secB2 from Francisella tularensis subsp. novicida exhibits a reduced biofilm formation, suggesting that both chaperone paralogs participate in the secretion of specific factors important for the attachment to abiotic surfaces (Margolis et al., 2010). Remarkably, the double $\sec B$ mutant was not viable, suggesting that in the case of F. tularensis, SecB chaperones have overlapping functions essential for bacterial survival (Margolis et al., 2010).

\section{CONCLUDING REMARKS}

Extensive genetic and biochemical analyses of SecB chaperone tasking have undoubtedly revealed its key cellular roles as part of the network of generic chaperones that orchestrate proteostasis in E. coli. The fact that SecB binds its substrates in a non-native state and prevents their unproductive folding is in agreement with its major role in delivering translocation competent proteins to the inner membrane, as observed for a large number of Sec-dependent presecretory proteins, for the ABC transporter substrate HasA, and perhaps for other proteins whose secretion relies on specific secretion systems that lack dedicated chaperones.

In contrast with the specific and well-described cooperative cascade between SecB and SecA during post-translational targeting of Sec-dependent precursors, the interplay between SecB, TF and DnaK remains poorly understood, and there is a clear lack of knowledge about the substrates that are shared between the three chaperones in vivo. In addition, it is not known whether these chaperones actively cooperate to facilitate export of certain proteins, both under normal and stress conditions, and to what extent such cooperation influences early partitioning of newly synthesized proteins. Similarly, it remains to be determined whether some cytosolic proteins or protein complexes do require SecB for their folding and/or assembly, as it is the case for the SecB-like protein Rv1957 and its TA system in M. tuberculosis. These are truly open questions that need to be addressed.

The relatively frequent association of SecB proteins with different TA families is intriguing and may reveal interesting new SecB functions, perhaps reflecting a link between toxin activation and membrane jamming. In this respect, an important mechanistic issue will be to determine how TA systems have acquired such a unique addiction for $\mathrm{SecB}$ chaperones. Finally, the sporadic presence of solitary SecB-like proteins in monoderm bacteria also suggests novel SecB functions to be discovered.

\section{ACKNOWLEDGMENTS}

We thank Petra Langendijk and Olivera Francetic for insightful discussion and Gwennaele Fichant's group for sharing their inhouse bacterial genome database. This work was supported by a French MENRT fellowship and an FRM grant (FDT20140930836) to Ambre Sala and a mycoTAC ANR grant (ANR-13-BSV8-001001) to Pierre Genevaux.

\section{REFERENCES}

Agashe, V. R., Guha, S., Chang, H. C., Genevaux, P., Hayer-Hartl, M., Stemp, M., et al. (2004). Function of trigger factor and DnaK in multidomain protein folding: increase in yield at the expense of folding speed. Cell 117, 199-209. doi: 10.1016/S0092-8674(04)00299-5

Altman, E., Kumamoto, C. A., and Emr, S. D. (1991). Heat-shock proteins can substitute for SecB function during protein export in Escherichia coli. EMBO J. 10, 239-245.

Baars, L., Ytterberg, A. J., Drew, D., Wagner, S., Thilo, C., Van Wijk, K. J., et al. (2006). Defining the role of the Escherichia coli chaperone SecB using comparative proteomics. J. Biol. Chem. 281, 10024-10034. doi: 10.1074/jbc.M509929200

Babu, M., Diaz-Mejia, J. J., Vlasblom, J., Gagarinova, A., Phanse, S., Graham, C., et al. (2011). Genetic interaction maps in Escherichia coli reveal functional crosstalk among cell envelope biogenesis pathways. PLoS Genet. 7:e1002377. doi: 10.1371/journal.pgen.1002377

Bechtluft, P., Nouwen, N., Tans, S. J., and Driessen, A. J. M. (2010). SecB-a chaperone dedicated to protein translocation. Mol. Biosyst. 6, 620-627. doi: $10.1039 / \mathrm{b} 915435 \mathrm{c}$ 
Bechtluft, P., Van Leeuwen, R. G. H., Tyreman, M., Tomkiewicz, D., Nouwen, N., Tepper, H. L., et al. (2007). Direct observation of chaperone-induced changes in a protein folding pathway. Science 318, 1458-1461. doi: 10.1126/science. 1144972

Bordes, P., Cirinesi, A. M., Ummels, R., Sala, A., Sakr, S., Bitter, W., et al. (2011). SecB-like chaperone controls a toxin-antitoxin stress-responsive system in Mycobacterium tuberculosis. Proc. Natl. Acad. Sci. U.S.A. 108, 8438-8443. doi 10.1073/pnas.1101189108

Brehmer, D., Rudiger, S., Gassler, C. S., Klostermeier, D., Packschies, L., Reinstein, J., et al. (2001). Tuning of chaperone activity of Hsp70 proteins by modulation of nucleotide exchange. Nat. Struct. Biol. 8, 427-432. doi: 10.1038/87588

Bukau, B., Deuerling, E., Pfund, C., and Craig, E. A. (2000). Getting newly synthesized proteins into shape. Cell 101, 119-122. doi: 10.1016/S0092-8674(00) 80806-5

Calloni, G., Chen, T., Schermann, S. M., Chang, H. C., Genevaux, P., Agostini, F., et al. (2012). DnaK functions as a central hub in the E. coli chaperone network. Cell Rep. 1, 251-264. doi: 10.1016/j.celrep.2011.12.007

Castanie-Cornet, M. P., Bruel, N., and Genevaux, P. (2014). Chaperone networking facilitates protein targeting to the bacterial cytoplasmic membrane. Biochim. Biophys. Acta 1843, 1442-1456. doi: 10.1016/j.bbamcr.2013.11.007

Chatzi, K. E., Sardis, M. F., Karamanou, S., and Economou, A. (2013). Breaking on through to the other side: protein export through the bacterial Sec system. Biochem. J. 449, 25-37. doi: 10.1042/BJ20121227

Collier, D. N., Bankaitis, V. A., Weiss, J. B., and Bassford, P. J. Jr. (1988). The antifolding activity of $\mathrm{SecB}$ promotes the export of the E. coli maltose-binding protein. Cell 53, 273-283. doi: 10.1016/0092-8674(88)90389-3

Crane, J. M., Mao, C. F., Lilly, A. A., Smith, V. F., Suo, Y. Y., Hubbell, W. L., et al (2005). Mapping of the docking of SecA onto the chaperone SecB by site-directed spin labeling: insight into the mechanism of ligand transfer during protein export. J. Mol. Biol. 353, 295-307. doi: 10.1016/j.jmb.2005.08.022

Crane, J. M., Suo, Y. Y., Lilly, A. A., Mao, C. F., Hubbell, W. L., and Randall, L. L. (2006). Sites of interaction of a precursor polypeptide on the export chaperone SecB mapped by site-directed spin labeling. J. Mol. Biol. 363, 63-74. doi: 10.1016/j.jmb.2006.07.021

Crooke, E., Brundage, L., Rice, M., and Wickner, W. (1988a). ProOmpA spontaneously folds in a membrane assembly competent state which trigger factor stabilizes. EMBO J. 7, 1831-1835.

Crooke, E., Guthrie, B., Lecker, S., Lill, R., and Wickner, W. (1988b). ProOmpA is stabilized for membrane translocation by either purified E. coli trigger factor or canine signal recognition particle. Cell 54, 1003-1011. doi: 10.1016/00928674(88)90115-8

Crooke, E., and Wickner, W. (1987). Trigger factor: a soluble protein that folds proOmpA into a membrane-assembly-competent form. Proc. Natl. Acad. Sci. U.S.A 84, 5216-5220. doi: 10.1073/pnas.84.15.5216

Dalbey, R. E., Wang, P., and Kuhn, A. (2011). Assembly of bacterial inner membrane proteins. Annu. Rev. Biochem. 80, 161-187. doi: 10.1146/annurev-biochem060409-092524

Danese, P. N., Murphy, C. K., and Silhavy, T. J. (1995). Multicopy suppression of cold-sensitive sec mutations in Escherichia coli. J. Bacteriol. 177, 4969-4973.

Debarbieux, L., and Wandersman, C. (2001). Folded HasA inhibits its own secretion through its ABC exporter. EMBO J. 20, 4657-4663. doi: 10.1093/emboj/20.17.4657

Dekker, C., De Kruijff, B., and Gros, P. (2003). Crystal structure of SecB from Escherichia coli. J. Struct. Biol. 144, 313-319. doi: 10.1016/j.jsb.2003.09.012

Delepelaire, P. (2004). Type I secretion in gram-negative bacteria. Biochim. Biophys Acta 1694, 149-161. doi: 10.1016/j.bbamcr.2004.05.001

Delepelaire, P., and Wandersman, C. (1998). The SecB chaperone is involved in the secretion of the Serratia marcescens HasA protein through an ABC transporter. EMBO J. 17, 936-944. doi: 10.1093/emboj/17.4.936

den Blaauwen, T., Terpetschnig, E., Lakowicz, J. R., and Driessen, A. J. (1997). Interaction of SecB with soluble SecA. FEBS Lett. 416, 35-38. doi: 10.1016/S00145793(97)01142-3

Derman, A. I., Puziss, J. W., Bassford, P. J. Jr., and Beckwith, J. (1993). A signa sequence is not required for protein export in prlA mutants of Escherichia coli. EMBO J. 12, 879-888.

Deuerling, E., Schulze-Specking, A., Tomoyasu, T., Mogk, A., and Bukau, B. (1999) Trigger factor and DnaK cooperate in folding of newly synthesized proteins. Nature 400, 693-696. doi: 10.1038/23301 du Plessis, D. J., Nouwen, N., and Driessen, A. J. (2011). The Sec translocase. Biochim. Biophys. Acta 1808, 851-865. doi: 10.1016/j.bbamem.2010.08.016

Fekkes, P., De Wit, J. G., Boorsma, A., Friesen, R. H., and Driessen, A. J. (1999). Zinc stabilizes the SecB binding site of SecA. Biochemistry 38, 5111-5116. doi: 10.1021/bi982818r

Fekkes, P., De Wit, J. G., Van Der Wolk, J. P., Kimsey, H. H., Kumamoto, C. A., and Driessen, A. J. (1998). Preprotein transfer to the Escherichia coli translocase requires the co-operative binding of SecB and the signal sequence to SecA. Mol. Microbiol. 29, 1179-1190. doi: 10.1046/j.1365-2958.1998.00997.x

Fekkes, P., Van Der Does, C., and Driessen, A. J. (1997). The molecular chaperone $\mathrm{SecB}$ is released from the carboxy-terminus of SecA during initiation of precursor protein translocation. EMBO J. 16, 6105-6113. doi: 10.1093/emboj/16. 20.6105

Ferbitz, L., Maier, T., Patzelt, H., Bukau, B., Deuerling, E., and Ban, N. (2004). Trigger factor in complex with the ribosome forms a molecular cradle for nascent proteins. Nature 431, 590-596. doi: 10.1038/nature02899

Francetic, O., Hanson, M. P., and Kumamoto, C. A. (1993). PrlA suppression of defective export of maltose-binding protein in SecB mutants of Escherichia coli. J. Bacteriol. 175, 4036-4044.

Gannon, P. M., and Kumamoto, C. A. (1993). Mutations of the molecular chaperone protein SecB which alter the interaction between Secb and maltose-binding protein. J. Biol. Chem. 268, 1590-1595.

Gannon, P. M., Li, P., and Kumamoto, C. A. (1989). The mature portion of Escherichia coli maltose-binding protein (MBP) determines the dependence of MBP on SecB for export. J. Bacteriol. 171, 813-818.

Genevaux, P., Georgopoulos, C., and Kelley, W. L. (2007). The Hsp70 chaperone machines of Escherichia coli: a paradigm for the repartition of chaperone functions. Mol. Microbiol. 66, 840-857. doi: 10.1111/j.1365-2958.2007. 05961.x

Genevaux, P., Keppel, F., Schwager, F., Langendijk-Genevaux, P. S., Hartl, F. U., and Georgopoulos, C. (2004). In vivo analysis of the overlapping functions of DnaK and trigger factor. EMBO Rep. 5, 195-200. doi: 10.1038/sj.embor.7400067

Gerdes, K., Christensen, S. K., and Lobner-Olesen, A. (2005). Prokaryotic toxin-antitoxin stress response loci. Nat. Rev. Microbiol. 3, 371-382. doi: 10.1038/nrmicro1147

Goeders, N., and Van Melderen, L. (2014). Toxin-antitoxin systems as multilevel interaction systems. Toxins (Basel) 6, 304-324. doi: 10.3390/toxins6010304

Gouridis, G., Karamanou, S., Gelis, I., Kalodimos, C. G., and Economou, A. (2009). Signal peptides are allosteric activators of the protein translocase. Nature 462, 363-367. doi: 10.1038/nature08559

Grabowicz, M., Yeh, J., and Silhavy, T. J. (2013). Dominant negative lptE mutation that supports a role for LptE as a plug in the LptD barrel. J. Bacteriol. 195, 1327-1334. doi: 10.1128/JB.02142-12

Gupta, A. (2009). Killing activity and rescue function of genome-wide toxinantitoxin loci of Mycobacterium tuberculosis. FEMS Microbiol. Lett. 290, 45-53. doi: 10.1111/j.1574-6968.2008.01400.x

Guthrie, B., and Wickner, W. (1990). Trigger factor depletion or overproduction causes defective cell division but does not block protein export. J. Bacteriol. 172, 5555-5562.

Hardy, S. J., and Randall, L. L. (1991). A kinetic partitioning model of selective binding of nonnative proteins by the bacterial chaperone SecB. Science 251, 439443. doi: $10.1126 /$ science. 1989077

Harrison, C. J., Hayer-Hartl, M., Di Liberto, M., Hartl, F., and Kuriyan, J. (1997). Crystal structure of the nucleotide exchange factor GrpE bound to the ATPase domain of the molecular chaperone DnaK. Science 276, 431-435. doi: $10.1126 /$ science. 276.5311 .431

Hartl, F. U., Lecker, S., Schiebel, E., Hendrick, J. P., and Wickner, W. (1990). The binding cascade of SecB to SecA to SecY/E mediates preprotein targeting to the E. coli plasma membrane. Cell 63, 269-279. doi: 10.1016/0092-8674(90)90160-G

Hayashi, S., and Wu, H. C. (1985). Accumulation of prolipoprotein in Escherichia coli mutants defective in protein secretion. J. Bacteriol. 161, 949-954.

Hoffmann, A., Becker, A. H., Zachmann-Brand, B., Deuerling, E., Bukau, B., and Kramer, G. (2012). Concerted action of the ribosome and the associated chaperone trigger factor confines nascent polypeptide folding. Mol. Cell 48, 63-74. doi: 10.1016/j.molcel.2012.07.018

Hoffmann, A., Bukau, B., and Kramer, G. (2010). Structure and function of the molecular chaperone Trigger Factor. Biochim. Biophys. Acta 1803, 650-661. doi: 10.1016/j.bbamcr.2010.01.017 
Holland, I. B., Schmitt, L., and Young, J. (2005). Type 1 protein secretion in bacteria, the ABC-transporter dependent pathway (review). Mol. Membr. Biol. 22, 29-39. doi: 10.1080/09687860500042013

Huber, D., Rajagopalan, N., Preissler, S., Rocco, M. A., Merz, F., Kramer, G., etal. (2011). SecA interacts with ribosomes in order to facilitate posttranslational translocation in bacteria. Mol. Cell 41, 343-353. doi: 10.1016/j.molcel.2010.12.028

Jin, T., and Inouye, M. (1995). Identification of the genes in multicopy plasmids affecting ompC and ompF expression in Escherichia coli. FEMS Microbiol. Lett. 133, 225-231. doi: 10.1111/j.1574-6968.1995.tb07889.x

Kaiser, C. M., Chang, H. C., Agashe, V. R., Lakshmipathy, S. K., Etchells, S. A., Hayer-Hartl, M., et al. (2006). Real-time observation of trigger factor function on translating ribosomes. Nature 444, 455-460. doi: 10.1038/nature 05225

Karamyshev, A. L., and Johnson, A. E. (2005). Selective SecA association with signal sequences in ribosome-bound nascent chains: a potential role for SecA in ribosome targeting to the bacterial membrane. J. Biol. Chem. 280, 37930-37940. doi: 10.1074/jbc.M509100200

Kerner, M. J., Naylor, D. J., Ishihama, Y., Maier, T., Chang, H. C., Stines, A. P. et al. (2005). Proteome-wide analysis of chaperonin-dependent protein folding in Escherichia coli. Cell 122, 209-220. doi: 10.1016/j.cell.2005.05.028

Khisty, V. J., Munske, G. R., and Randall, L. L. (1995). Mapping of the binding frame for the chaperone SecB within a natural ligand, galactose-binding protein. J. Biol. Chem. 270, 25920-25927. doi: 10.1074/jbc.270.43.25920

Kim, Y. E., Hipp, M. S., Bracher, A., Hayer-Hartl, M., and Hartl, F. U. (2013). Molecular chaperone functions in protein folding and proteostasis. Annu. Rev Biochem. 82, 323-355. doi: 10.1146/annurev-biochem-060208-092442

Kimsey, H. H., Dagarag, M. D., and Kumamoto, C. A. (1995). Diverse effects of mutation on the activity of the Escherichia coli export chaperone SecB. J. Biol. Chem. 270, 22831-22835. doi: 10.1074/jbc.270.39.22831

Knoblauch, N. T., Rudiger, S., Schonfeld, H. J., Driessen, A. J., Schneider-Mergener, J., and Bukau, B. (1999). Substrate specificity of the SecB chaperone. J. Biol. Chem. 274, 34219-34225. doi: 10.1074/jbc.274.48.34219

Kramer, G., Boehringer, D., Ban, N., and Bukau, B. (2009). The ribosome as a platform for co-translational processing, folding and targeting of newly synthesized proteins. Nat. Struct. Mol. Biol. 16, 589-597. doi: 10.1038/nsmb.1614

Kramer, G., Rauch, T., Rist, W., Vorderwulbecke, S., Patzelt, H., Schulze-Specking, A., et al. (2002). L23 protein functions as a chaperone docking site on the ribosome. Nature 419, 171-174. doi: 10.1038/nature01047

Kumamoto, C. A., and Beckwith, J. (1983). Mutations in a new gene, secB, cause defective protein localization in Escherichia coli. J. Bacteriol. 154, 253-260.

Kumamoto, C. A., and Beckwith, J. (1985). Evidence for specificity at an early step in protein export in Escherichia coli. J. Bacteriol. 163, 267-274.

Kusters, I., Van Den Bogaart, G., Kedrov, A., Krasnikov, V., Fulyani, F., Poolman, B., et al. (2011). Quaternary structure of SecA in solution and bound to SecYEG probed at the single molecule level. Structure 19, 430-439. doi 10.1016/j.str.2010.12.016

Kusters, R., De Vrije, T., Breukink, E., and De Kruijff, B. (1989). SecB protein stabilizes a translocation-competent state of purified prePhoE protein. J. Biol. Chem. 264, 20827-20830.

Kusukawa, N., Yura, T., Ueguchi, C., Akiyama, Y., and Ito, K. (1989). Effects of mutations in heat-shock genes groES and groEL on protein export in Escherichia coli. EMBO J. 8, 3517-3521.

Laminet, A. A., Kumamoto, C. A., and Pluckthun, A. (1991). Folding in vitro and transport in vivo of pre-Beta-Lactamase are SecB independent. Mol. Microbiol. 5 117-122. doi: 10.1111/j.1365-2958.1991.tb01832.x

Lecker, S., Lill, R., Ziegelhoffer, T., Georgopoulos, C., Bassford, P. J., Kumamoto, C. A., et al. (1989). Three pure chaperone proteins of Escherichia coli SecB, trigger factor and groel-form soluble complexes with precursor proteins in vitro. EMBO J. 8, 2703-2709.

Lee, H. C., and Bernstein, H. D. (2002). Trigger factor retards protein export in Escherichia coli. J. Biol. Chem. 277, 43527-43535. doi: 10.1074/jbc.M205950200

Letoffe, S., Ghigo, J. M., and Wandersman, C. (1994). Secretion of the Serratia marcescens HasA protein by an ABC transporter. J. Bacteriol. 176, 5372-5377.

Lewis, K. (2010). Persister cells. Annu. Rev. Microbiol. 64, 357-372. doi: 10.1146/annurev.micro.112408.134306

Liberek, K., Marszalek, J., Ang, D., Georgopoulos, C., and Zylicz, M. (1991). Escherichia coli DnaJ and GrpE heat shock proteins jointly stimulate
ATPase activity of DnaK. Proc. Natl. Acad. Sci. U.S.A. 88, 2874-2878. doi: 10.1073/pnas.88.7.2874

Lill, R., Crooke, E., Guthrie, B., and Wickner, W. (1988). The "trigger factor cycle" includes ribosomes, presecretory proteins, and the plasma membrane. Cell 54, 1013-1018. doi: 10.1016/0092-8674(88)90116-X

Liu, G., Topping, T. B., and Randall, L. L. (1989). Physiological role during export for the retardation of folding by the leader peptide of maltose-binding protein. Proc. Natl. Acad. Sci. U.S.A. 86, 9213-9217. doi: 10.1073/pnas.86.23.9213

Luirink, J., and Sinning, I. (2004). SRP-mediated protein targeting: structure and function revisited. Biochim. Biophys. Acta 1694, 17-35. doi: 10.1016/j.bbamcr.2004.03.013

Mah, N., Perez-Iratxeta, C., and Andrade-Navarro, M. A. (2010). Outer membrane pore protein prediction in mycobacteria using genomic comparison. Microbiology 156, 2506-2515. doi: 10.1099/mic.0.040089-0

Makarova, K. S., Wolf, Y. I., and Koonin, E. V. (2009). Comprehensive comparativegenomic analysis of type 2 toxin-antitoxin systems and related mobile stress response systems in prokaryotes. Biol. Direct 4, 19. doi: 10.1186/17456150-4-19

Marani, P., Wagner, S., Baars, L., Genevaux, P., De Gier, J. W., Nilsson, I., et al. (2006). New Escherichia coli outer membrane proteins identified through prediction and experimental verification. Protein Sci. 15, 884-889. doi: 10.1110/ps.051889506

Margolis, J. J., El-Etr, S., Joubert, L. M., Moore, E., Robison, R., Rasley, A., et al. (2010). Contributions of Francisella tularensis subsp. novicida chitinases and Sec secretion system to biofilm formation on chitin. Appl. Environ. Microbiol. 76, 596-608. doi: 10.1128/AEM.02037-09

Mcfarland, L., Francetic, O., and Kumamoto, C. A. (1993). A mutation of Escherichia coli SecA protein that partially compensates for the absence of SecB. J. Bacteriol. 175, 2255-2262.

Miller, A., Wang, L., and Kendall, D. A. (2002). SecB modulates the nucleotidebound state of SecA and stimulates ATPase activity. Biochemistry 41, 5325-5332. doi: 10.1021/bi025639p

Muller, J. P. (1996). Influence of impaired chaperone or secretion function on SecB production in Escherichia coli. J. Bacteriol. 178, 6097-6104.

Muren, E. M., Suciu, D., Topping, T. B., Kumamoto, C. A., and Randall, L. L. (1999). Mutational alterations in the homotetrameric chaperone SecB that implicate the structure as dimer of dimers. J. Biol. Chem. 274, 19397-19402. doi: 10.1074/jbc.274.27.19397

Nichols, R. J., Sen, S., Choo, Y. J., Beltrao, P., Zietek, M., Chaba, R., et al. (2011). Phenotypic landscape of a bacterial cell. Cell 144, 143-156. doi: 10.1016/j.cell.2010.11.052

O’Brien, E. P., Christodoulou, J., Vendruscolo, M., and Dobson, C. M. (2012). Trigger factor slows co-translational folding through kinetic trapping while sterically protecting the nascent chain from aberrant cytosolic interactions. J. Am. Chem. Soc. 134, 10920-10932. doi: 10.1021/ja302305u

Oh, E., Becker, A. H., Sandikci, A., Huber, D., Chaba, R., Gloge, F., et al. (2011). Selective ribosome profiling reveals the cotranslational chaperone action of trigger factor in vivo. Cell 147, 1295-1308. doi: 10.1016/j.cell.2011.10.044

Palmer, T., and Berks, B. C. (2012). The twin-arginine translocation (Tat) protein export pathway. Nat. Rev. Microbiol. 10, 483-496. doi: 10.1038/nrmicro2814

Patel, R., Smith, S. M., and Robinson, C. (2014). Protein transport by the bacterial Tat pathway. Biochim. Biophys. Acta 1843, 1620-1628. doi: 10.1016/j.bbamcr.2014.02.013

Phillips, G. J., and Silhavy, T. J. (1990). Heat-shock proteins DnaK and GroEL facilitate export of LacZ hybrid proteins in E. coli. Nature 344, 882-884. doi: $10.1038 / 344882 \mathrm{a} 0$

Powers, E. L., and Randall, L. L. (1995). Export of periplasmic galactose-binding protein in Escherichia coli depends on the chaperone SecB. J. Bacteriol. 177, 19061907.

Qi, H. Y., Hyndman, J. B., and Bernstein, H. D. (2002). DnaK promotes the selective export of outer membrane protein precursors in SecA-deficient Escherichia coli. J. Biol. Chem. 277, 51077-51083. doi: 10.1074/jbc.M209238200

Raine, A., Lovmar, M., Wikberg, J., and Ehrenberg, M. (2006). Trigger factor binding to ribosomes with nascent peptide chains of varying lengths and sequences. J. Biol. Chem. 281, 28033-28038. doi: 10.1074/jbc.M605753200

Ramage, H. R., Connolly, L. E., and Cox, J. S. (2009). Comprehensive functional analysis of Mycobacterium tuberculosis toxin-antitoxin systems: implications for pathogenesis, stress responses, and evolution. PLoS Genet. 5:e1000767. doi: 10.1371/journal.pgen. 1000767 
Randall, L. L., Crane, J. M., Lilly, A. A., Liu, G. P., Mao, C. F., Patel, C. N., et al. (2005). Asymmetric binding between SecA and SecB two symmetric proteins: implications for function in export. J. Mol. Biol. 348, 479-489. doi: 10.1016/j.jmb.2005.02.036

Randall, L. L., Crane, J. M., Liu, G., and Hardy, S. J. (2004). Sites of interaction between SecA and the chaperone SecB, two proteins involved in export. Protein Sci. 13, 1124-1133. doi: 10.1110/ps.03410104

Randall, L. L., and Hardy, S. J. (1995). High selectivity with low specificity: how SecB has solved the paradox of chaperone binding. Trends Biochem. Sci. 20, 65-69. doi: 10.1016/S0968-0004(00)88959-8

Randall, L. L., and Hardy, S. J. (2002). SecB, one small chaperone in the complex milieu of the cell. Cell. Mol. Life Sci. 59, 1617-1623. doi: 10.1007/PL00012488

Rigel, N. W., Gibbons, H. S., Mccann, J. R., Mcdonough, J. A., Kurtz, S., and Braunstein, M. (2009). The accessory SecA2 system of mycobacteria requires ATP binding and the canonical SecAl. J. Biol. Chem. 284, 9927-9936. doi: 10.1074/jbc.M900325200

Rudiger, S., Germeroth, L., Schneider-Mergener, J., and Bukau, B. (1997). Substrate specificity of the DnaK chaperone determined by screening cellulose-bound peptide libraries. EMBO J. 16, 1501-1507. doi: 10.1093/emboj/16.7.1501

Saibil, H. R., Fenton, W. A., Clare, D. K., and Horwich, A. L. (2013). Structure and allostery of the chaperonin GroEL. J. Mol. Biol. 425, 1476-1487. doi: 10.1016/j.jmb.2012.11.028

Saio, T., Guan, X., Rossi, P., Economou, A., and Kalodimos, C. G. (2014). Structural basis for protein antiaggregation activity of the trigger factor chaperone. Science 344:1250494. doi: 10.1126/science.1250494

Sakr, S., Cirinesi, A. M., Ullers, R. S., Schwager, F., Georgopoulos, C., and Genevaux P. (2010). Lon protease quality control of presecretory proteins in Escherichia col and its dependence on the SecB and DnaJ (Hsp40) chaperones. J. Biol. Chem. 285, 23504-23512. doi: 10.1074/jbc.M110.133058

Sala, A., Bordes, P., Fichant, G., and Genevaux, P. (2013a). "Toxin-antitoxin loci in Mycobacterium tuberculosis," in Prokaryotic Toxin-Antitoxins, ed. K. Gerdes (Berlin: Springer), 295-314.

Sala, A., Calderon, V., Bordes, P., and Genevaux, P. (2013b). TAC from Mycobacterium tuberculosis: a paradigm for stress-responsive toxin-antitoxin systems controlled by SecB-like chaperones. Cell Stress Chaperones 18, 129-135. doi: 10.1007/s12192-012-0396-5

Sapriel, G., Wandersman, C., and Delepelaire, P. (2002). The N terminus of the HasA protein and the SecB chaperone cooperate in the efficient targeting and secretion of HasA via the ATP-binding cassette transporter. J. Biol. Chem. 277, 6726-6732. doi: 10.1074/jbc.M108632200

Sapriel, G., Wandersman, C., and Delepelaire, P. (2003). The SecB chaperone is bifunctional in Serratia marcescens: SecB is involved in the Sec pathway and required for HasA secretion by the $\mathrm{ABC}$ transporter. J. Bacteriol. 185, 80-88. doi: 10.1128/JB.185.1.80-88.2003

Saraogi, I., and Shan, S. O. (2014). Co-translational protein targeting to the bacterial membrane. Biochim. Biophys. Acta 1843, 1433-1441. doi: 10.1016/j.bbamcr.2013.10.013

Schuessler, D. L., Cortes, T., Fivian-Hughes, A. S., Lougheed, K. E., Harvey, E., Buxton, R. S., et al. (2013). Induced ectopic expression of HigB toxin in Mycobacterium tuberculosis results in growth inhibition, reduced abundance of a subset of mRNAs and cleavage of tmRNA. Mol. Microbiol. 90, 195-207. doi: $10.1111 / \mathrm{mmi} .12358$

Shimizu, H., Nishiyama, K., and Tokuda, H. (1997). Expression of gpsA encoding biosynthetic sn-glycerol 3-phosphate dehydrogenase suppresses both the LBphenotype of a secB null mutant and the cold-sensitive phenotype of a secG null mutant. Mol. Microbiol. 26, 1013-1021. doi: 10.1046/j.1365-2958.1997.6392003.x

Singh, R., Kraft, C., Jaiswal, R., Sejwal, K., Kasaragod, V. B., Kuper, J., et al. (2014). Cryo-electron microscopic structure of SecA protein bound to the $70 \mathrm{~S}$ ribosome. J. Biol. Chem. 289, 7190-7199.

Snyder, W. B., and Silhavy, T. J. (1992). Enhanced export of beta-galactosidase fusion proteins in prlF mutants is Lon dependent. J. Bacteriol. 174, 5661-5668.

Sullivan, J. T., Young, E. F., Mccann, J. R., and Braunstein, M. (2012). The Mycobacterium tuberculosis SecA2 system subverts phagosome maturation to promote growth in macrophages. Infect. Immun. 80, 996-1006. doi: 10.1128/IAI.05987-11

Teter, S. A., Houry, W. A., Ang, D., Tradler, T., Rockabrand, D., Fischer, G., et al. (1999). Polypeptide flux through bacterial Hsp70: DnaK cooperates with trigger factor in chaperoning nascent chains. Cell 97, 755-765. doi: 10.1016/S0092$8674(00) 80787-4$
Trun, N. J., and Silhavy, T. J. (1989). PrlC, a suppressor of signal sequence mutations in Escherichia coli, can direct the insertion of the signal sequence into the membrane. J. Mol. Biol. 205, 665-676. doi: 10.1016/0022-2836(89)90312-4

Trun, N. J., Stader, J., Lupas, A., Kumamoto, C., and Silhavy, T. J. (1988). Two cellular components, PrlA and $\mathrm{SecB}$, that recognize different sequence determinants are required for efficient protein export. J. Bacteriol. 170, 5928-5930.

Ullers, R. S., Ang, D., Schwager, F., Georgopoulos, C., and Genevaux, P. (2007). Trigger factor can antagonize both $\mathrm{SecB}$ and DnaK/DnaJ chaperone functions in Escherichia coli. Proc. Natl. Acad. Sci. U.S.A. 104, 3101-3106. doi: 10.1073/pnas.0608232104

Ullers, R. S., Luirink, J., Harms, N., Schwager, F., Georgopoulos, C., and Genevaux, P. (2004). SecB is a bona fide generalized chaperone in Escherichia coli. Proc. Natl. Acad. Sci. U.S.A. 101, 7583-7588. doi: 10.1073/pnas.0402398101

Valent, Q. A., Kendall, D. A., High, S., Kusters, R., Oudega, B., and Luirink, J. (1995). Early events in preprotein recognition in E. coli: interaction of SRP and trigger factor with nascent polypeptides. EMBO J. 14, 5494-5505.

van der Sluis, E. O., and Driessen, A. J. (2006). Stepwise evolution of the Sec machinery in Proteobacteria. Trends Microbiol. 14, 105-108. doi: 10.1016/j.tim.2006.01.009

Watanabe, T., Hayashi, S., and Wu, H. C. (1988). Synthesis and export of the outer membrane lipoprotein in Escherichia coli mutants defective in generalized protein export. J. Bacteriol. 170, 4001-4007.

Wei, S. Q., and Stader, J. (1994). A new suppressor of a lamB signal sequence mutation, prlZ1, maps to 69 minutes on the Escherichia coli chromosome. J. Bacteriol. 176, 5704-5710.

Wild, J., Altman, E., Yura, T., and Gross, C. A. (1992). DnaK and DnaJ heat shock proteins participate in protein export in Escherichia coli. Genes Dev. 6, 1165-1172. doi: 10.1101/gad.6.7.1165

Wild, J., Walter, W. A., Gross, C. A., and Altman, E. (1993). Accumulation of secretory protein precursors in Escherichia coli induces the heat shock response. J. Bacteriol. 175, 3992-3997.

Wolff, N., Sapriel, G., Bodenreider, C., Chaffotte, A., and Delepelaire, P. (2003). Antifolding activity of the $\mathrm{SecB}$ chaperone is essential for secretion of HasA, a quickly folding ABC pathway substrate. J. Biol. Chem. 278, 38247-38253. doi: 10.1074/jbc.M302322200

Woodbury, R. L., Topping, T. B., Diamond, D. L., Suciu, D., Kumamoto, C. A., Hardy, S. J., et al. (2000). Complexes between protein export chaperone SecB and SecA - Evidence for separate sites on SecA providing binding energy and regulatory interactions. J. Biol. Chem. 275, 24191-24198. doi: 10.1074/jbc. M002885200

Xu, Z., Knafels, J. D., and Yoshino, K. (2000). Crystal structure of the bacterial protein export chaperone SecB. Nat. Struct. Biol. 7, 1172-1177. doi: 10.1038/82040

Yamaguchi, Y., and Inouye, M. (2011). Regulation of growth and death in Escherichia coli by toxin-antitoxin systems. Nat. Rev. Microbiol. 9, 779-790. doi: $10.1038 /$ nrmicro2651

Zhou, J., and Xu, Z. (2003). Structural determinants of SecB recognition by SecA in bacterial protein translocation. Nat. Struct. Biol. 10, 942-947. doi: $10.1038 /$ nsb 980

Zuber, B., Chami, M., Houssin, C., Dubochet, J., Griffiths, G., and Daffe, M. (2008). Direct visualization of the outer membrane of mycobacteria and corynebacteria in their native state. J. Bacteriol. 190, 5672-5680. doi: 10.1128/JB.01919-07

Conflict of Interest Statement: The authors declare that the research was conducted in the absence of any commercial or financial relationships that could be construed as a potential conflict of interest.

Received: 25 September 2014; accepted: 17 November 2014; published online: 05 December 2014.

Citation: Sala A, Bordes P and Genevaux P (2014) Multitasking SecB chaperones in bacteria. Front. Microbiol. 5:666. doi: 10.3389/fmicb.2014.00666

This article was submitted to Microbial Physiology and Metabolism, a section of the journal Frontiers in Microbiology.

Copyright $\left({ }_{0} 2014\right.$ Sala, Bordes and Genevaux. This is an open-access article distributed under the terms of the Creative Commons Attribution License (CC BY). The use, distribution or reproduction in other forums is permitted, provided the original author(s) or licensor are credited and that the original publication in this journal is cited, in accordance with accepted academic practice. No use, distribution or reproduction is permitted which does not comply with these terms. 